\title{
GAUSS MAP OF MINIMAL SURFACES WITH RAMIFICATION
}

\author{
MIN RU
}

\begin{abstract}
We prove that for any complete minimal surface $M$ immersed in $R^{n}$, if in $C P^{n-1}$ there are $q>n(n+1) / 2$ hyperplanes $H_{j}$ in general position such that the Gauss map of $M$ is ramified over $H_{j}$ with multiplicity at least $e_{j}$ for each $j$ and
\end{abstract}

$$
\sum_{j=1}^{q}\left(1-\frac{(n-1)}{e_{j}}\right)>n(n+1) / 2,
$$

then $M$ must be flat.

\section{INTRODUCTION}

Let $x: M \rightarrow R^{n}$ be a (smooth, oriented) minimal surface immersed in $R^{n}$. Make $M$ into a Riemann surface by decreeing that the 1 -form $d \xi_{1}+i d \xi_{2}$ is of type $(1,0)$, where $\left(\xi_{1}, \xi_{2}\right)$ are any local isothermal coordinates of $M$. The Gauss map of $x$ is defined to be

$$
G: M \rightarrow Q_{n-2}(C) \subset C P^{n-1}, \quad G(z)=[(\partial x / \partial z)]
$$

where $[(\cdot)]$ denotes the complex line in $C^{n}$ through the origin and $(\cdot), z=$ $\xi_{1}+i \xi_{2}$ is the holomorphic coordinate of $M$, and

$$
Q_{n-2}(C)=\left\{\left(w_{0}: \cdots: w_{n-1} ; w_{0}^{2}+\cdots+w_{n-1}^{2}=0\right\} \subset C P^{n-1} .\right.
$$

By the assumption of minimality of $M, G$ is a holomorphic map of $M$ into $C P^{n-1}$. It is a natural question to study the "value distribution" properties of the Gauss map $G$. Fujimoto (see [8]) has shown that the Gauss map of a nonflat minimal surfaces can omit at most $n(n+1) / 2$ hyperplanes in general position in $C P^{n-1}$ under the assumption that $G$ is nondegenerate. The "nondegenerate" assumption was removed by the author (see [13]). The purpose of this paper is to study more general "value distribution" properties of the Gauss map. In particular, we study the Gauss map with ramification.

One says that $G$ is ramified over a hyperplane $H=\left\{[w] \in C P^{n-1}: a_{0} w_{0}+\right.$ $\left.\cdots+a_{n-1} w_{n-1}=0\right\}$ with multiplicity at least $e$ if all the zeros of the function $g_{H}=(G, A)$ have orders at least $e$, where $A=\left(a_{0}, \ldots, a_{n-1}\right)$. If the image of $G$ omits $H$, we shall say that $G$ is ramified over $H$ with multiplicity $\infty$.

Our main result is the following:

Received by the editors July 10, 1991.

1991 Mathematics Subject Classification. Primary 53A10, 32H30. 
Theorem 1. Let $M$ be a complete minimal surface immersed in $R^{n}$ and assume that the Gauss map $G$ of $M$ is $k$-nondegenerate (that is $G(M)$ is contained in a $k$-dimensional linear subspace of $C P^{n-1}$, but none of lower dimension), $1 \leq k \leq n-1$. Let $H_{i} \subset C P^{n-1}$ be $q$ hyperplanes in general position. If $G$ is ramified over $H_{i}$ with multiplicity at least $e_{i}$ for each $i$. Then

$$
\sum_{j=1}^{q}\left(1-\frac{k}{e_{j}}\right) \leq(k+1)\left(n-\frac{k}{2}-1\right)+n .
$$

In particular, for any complete minimal surface $M$ immersed in $R^{n}$, if in $C P^{n-1}$ there are $q>n(n+1) / 2$ hyperplanes in general position such that its Gauss map $G$ is ramified over $H_{j}$ with multiplicity at least $e_{j}$ for each $j$ and

$$
\sum_{j=1}^{q}\left(1-\frac{(n-1)}{e_{j}}\right)>n(n+1) / 2,
$$

then $M$ must be flat.

In the case $m=3, Q_{1}(C)$ can be identified with $C P^{1}$. We have a better result.

Theorem 2. Let $M$ be a complete minimal surface $\left(\subset R^{3}\right)$. If there are $q(q>4)$ distinct points $a_{1}, \ldots, a_{q} \in C P^{1}$ such that the Gauss map of $M$ is ramified over $a_{j}$ with multiplicity at least $e_{j}$ for each $j$. and $\sum_{j=1}^{q}\left(1-1 / e_{j}\right)>4$, then $M$ must be flat.

In particular, if the Gauss map omits five distinct points, then $M$ must be flat.

\section{FACTS ON HOLOMORPHIC CURVES INTO PROJECTIVE SPACES}

We shall recall some known results in the theory of holomorphic curves.

(A) Associated curve. Let $f$ be a nondegenerated holomorphic map of $\Delta_{R}$ : $\{z:|z|<R\}$ into $C P^{k}$, where $0<R \leq \infty$. Take a reduced representation $f=\left[Z_{0}: \cdots: Z_{k}\right]$, where $Z=\left(Z_{0}, \ldots, Z_{k}\right): \Delta_{R} \rightarrow C^{k+1}-\{0\}$. Denote by $Z^{(j)}$ the $j$ th derivative of $Z$ and define

$$
\Lambda_{j}=Z^{(0)} \wedge \cdots \wedge Z^{(j)}: \Delta_{R} \rightarrow \bigwedge^{j+1} C^{k+1}
$$

for $0 \leq j \leq k$. Evidently $\Lambda_{k+1} \equiv 0$.

let $P: \bigwedge^{j+1} C^{k+1}-\{0\} \rightarrow C P^{N_{j}}$ denote the canonical projection, where $N_{j}=\left(\begin{array}{c}k+1 \\ j+1\end{array}\right)-1$. The $j$ th associated curve of $f$ is the map $f_{j}=P\left(\Lambda_{j}\right)$.

It is well known [4] (also see [16]) that the pull-back $\Omega_{j}$ of the Fubini-study metric on $C P^{N_{j}}$ by $f_{j}$ is given by

$$
\Omega_{j}=d d^{c} \log \left|\Lambda_{j}\right|^{2}=\frac{i}{2 \pi} \frac{\left|\Lambda_{j-1}\right|^{2}\left|\Lambda_{j+1}\right|^{2}}{\left|\Lambda_{j}\right|^{4}} d z \wedge d \bar{z},
$$

for $0 \leq j \leq k$ and by convention $\Lambda_{-1} \equiv 1$. Note that $\Omega_{k} \equiv 0$. It follows that

$$
\operatorname{Ric} \Omega_{j}=\Omega_{j-1}+\Omega_{j+1}-2 \Omega_{j} .
$$


Take a hyperplane $H:(W, A)=0$, where $A=\left(a_{0}, \ldots, a_{k}\right)$ is a unit vector. Define

$$
\varphi_{j}(H)=\frac{\left|\Lambda_{j} \vee A\right|^{2}}{\left|\Lambda_{j}\right|^{2}|A|^{2}} .
$$

Note that $0 \leq \varphi_{j}(H) \leq \varphi_{j+1}(H) \leq 1$ for $0 \leq j \leq k$ and $\varphi_{k}(H)=1$.

We need the following well-known lemma (see [4, 16 and 17]).

Lemma 2.1. Let $H$ be a hyperplane in $C P^{k}$, then for any constant $N>1$, for $0 \leq p \leq k-1$,

$$
d d^{c} \log \frac{1}{N-\log \varphi_{p}(H)} \geq\left\{\frac{\varphi_{p+1}(H)}{\varphi_{p}(H)\left(N-\log \varphi_{p}(H)\right)^{2}}-\frac{1}{N}\right\} \Omega_{p},
$$

on $\Delta_{R}-\left\{\varphi_{p}=0\right\}$.

(B) Nochka weights and product to sum estimate. We consider $q$ hyperplanes $H_{j} \quad(1 \leq j \leq q)$ in $C P^{k}$ which are given by $H_{j}:\left(W, A_{j}\right)=0$. According to Chen [2], we give the following definition.

Definition 2.2. We say that hyperplanes $H_{1}, \ldots, H_{q}$ are in $n$-subgeneral position if, for every $1 \leq j_{0}<\cdots<j_{n} \leq q, A_{j_{0}}, A_{j_{1}}, \ldots, A_{j_{n}}$ generate $C^{k+1}$.

In [11] (see also [2]), Nochka has given the following lemma to prove the Cartan conjecture.

Lemma 2.3. Let $H_{1}, \ldots, H_{q}$ be hyperplanes in $C P^{k}$ located in the $n$-subgeneral position, where $q>2 n-k+1$. Then there are some constants $\omega(1), \ldots, \omega(q)$ and $\theta$ satisfying the following condition:

(i) $0<\omega(j) \theta \leq 1 \quad(1 \leq j \leq q)$,

(ii) $\theta\left(\sum_{j=1}^{q} \omega(j)-k-1\right)=q-2 n+k-1$,

(iii) $1 \leq(n+1) /(k+1) \leq \theta \leq(2 n-k+1) /(k+1)$,

(iv) if $R \subset Q$ and $0<\# R \leq n+1$, then $\sum_{j \in R} \omega(j) \leq d(R)$.

For the proof, see [2] or [11].

Definition 2.4. We call constants $\omega(j) \quad(1 \leq j \leq q)$ and $\theta$ above Nochka weights and a Nochka constant for $H_{1}, \ldots, H_{q}$ respectively.

Nachka weights are useful because of the following lemma.

Lemma 2.5. Under the above assumptions. Let $E_{1}, \ldots, E_{q}$ be a sequence of real numbers with $E_{j} \geq 1$ for all $j$. Then for any subset $B$ of the set $\{1,2, \ldots, q\}$ with $0<\# B \leq n+1$, there exists a subset $C$ of $B$ such that $\left\{A_{j} \mid j \in C\right\}$ is $a$ base of the linear space spanned by $\left\{A_{j} \mid j \in B\right\}$ and

$$
\prod_{j \in B} E_{j}^{\omega(j)} \leq \prod_{j \in C} E_{j}
$$

where $\omega(j)$ are the Nochka weights associated to hyperplanes $H_{j}:\left(A_{j}, W\right)=0$, $j=1,2, \ldots, q$.

For the proof, see [2] or [11].

We also have the following product to sum estimate. 
Lemma 2.6 (see Chen [2]). Under the above assumptions. For $0 \leq p \leq k-1$, any constant $N>1,1 / q \leq \lambda_{p} \leq 1 /(k-p)$, there exists a positive constant $c_{p}>0$ only depends on $p$ and the given hyperplanes such that

$$
\begin{gathered}
c_{p} \prod_{j=1}^{q}\left(\frac{\varphi_{p+1}\left(H_{j}\right)^{\omega(j)}}{\varphi_{p}\left(H_{j}\right)} \frac{1}{\left(N-\log \varphi_{p}\left(H_{j}\right)\right)^{2}}\right)^{\lambda_{p}} \\
\quad \leq \sum_{j=1}^{q} \frac{\varphi_{p+1}\left(H_{j}\right)}{\varphi_{p}\left(H_{j}\right)\left(N-\log \varphi_{p}\left(H_{j}\right)\right)^{2}},
\end{gathered}
$$

on $\Delta_{R}-\left\{\varphi_{p}=0\right\}$

\section{Metrics With Negative CURVATURe}

We retain the notation of the last section. Let $f: \Delta_{R} \rightarrow C P^{k}$ be a nondegenerate holomorphic map. Take a reduced representation $f=\left[Z_{0}: \cdots: Z_{k}\right]$ where $Z=\left(Z_{0}, \ldots, Z_{k}\right): \Delta_{R} \rightarrow C^{k+1}-\{0\}$ is a holomorphic map. Let $H_{1}, \ldots, H_{q}$ be hyperplanes in $C P^{k}$ located in $n$-subgeneral position. Let $\omega(j)$ be their Nochka weights.

Let $f$ be ramified over $H_{j}$ with multiplicity at least $e_{j}$ for each $j$. Assume that

$$
\sum_{j=1}^{q}\left(1-\frac{k}{e_{j}}\right)>2 n-k+1,
$$

we shall construct a continuous pseudo-metric on $\Delta_{R}$ such that its Gauss curvature is less than or equal to -1 . So that we can use Schwarz lemma to obtain our main inequality.

Let $\Omega_{p}=\frac{i}{2 \pi} h_{p}(z) d z \wedge d \bar{z}$. Let

$$
\sigma_{p}=c_{p} \prod_{j=1}^{q}\left[\left(\frac{\varphi_{p+1}\left(H_{j}\right)}{\varphi_{p}\left(H_{j}\right)}\right)^{\omega(j)\left(1-k / e_{j}\right)} \frac{1}{\left(N-\log \varphi_{p}\left(H_{j}\right)\right)^{2}}\right]^{\lambda_{p}} h_{p} .
$$

Where $c_{p}$ is the constant in the product to sum estimate,

$$
\lambda_{p}=1 /\left((k-p)+(k-p)^{2} \frac{2 q}{N}\right),
$$

and $N>1$.

We take the geometric mean of the $\sigma_{p}$ and define

$$
\Gamma=\frac{i}{2 \pi} c \prod_{p=0}^{k-1} \sigma_{p}^{\beta_{k} / \lambda_{p}} d z \wedge d \bar{z} .
$$

where $\beta_{k}=1 /\left(\sum_{p=0}^{k-1} \lambda_{p}^{-1}\right)$, and $c=2\left(\prod_{p=0}^{k-1} \lambda_{p}^{\lambda_{p}^{-1}}\right)^{\beta_{k}}$.

Let

$$
\Gamma=\frac{i}{2 \pi} h(z) d z \wedge d \bar{z} .
$$

We now compute $h(z)$. By (3.1) and (3.2), we have

$$
h(z)=c\left[\prod_{j=1}^{q} \frac{k}{\varphi_{0}\left(H_{j}\right)^{\omega(j)\left(1-1 / e_{j}\right) \beta_{k}}} \prod_{p=0}^{k=1} \frac{h_{p}^{\beta_{k} / \lambda_{p}}}{\left(N-\log \varphi_{p}\left(H_{j}\right)\right)}\right] .
$$


By (2.1),

$$
h_{p}^{1 / \lambda_{p}}=\left(\frac{\left|\Lambda_{p-1}\right|^{2}\left|\Lambda_{p+1}\right|^{2}}{\left|\Lambda_{p}\right|^{4}}\right)^{(k-p)+(k-p)^{2} 2 q / N}
$$

so

$$
\prod_{p=0}^{k-1} h_{p}^{1 / \lambda_{p}}=\left|\Lambda_{0}\right|^{-2(k+1)-\left(k^{2}+2 k-1\right) 4 q / N}\left|\Lambda_{1}\right|^{8 q / N} \cdots\left|\Lambda_{k-1}\right|^{8 q / N}\left|\Lambda_{k}\right|^{2+4 q / N} .
$$

Notice that $\left|\Lambda_{0}\right|=|Z|$, and $\varphi_{0}\left(H_{j}\right)=\left|\left(Z, A_{j}\right)\right|^{2} /|Z|^{2}$, therefore

$$
h(z)=c\left[\frac{|Z|^{\sum_{j=1}^{q} \omega(j)\left(1-k / e_{j}\right)-(k+1)-\left(k^{2}+2 k-1\right) 2 q / N}\left(\left|\Lambda_{1}\right| \cdots\left|\Lambda_{k-1}\right|\right)^{4 q / N}\left|\Lambda_{k}\right|^{1+2 q / N}}{\prod_{j=1}^{q}\left|\left(Z, A_{j}\right)\right|^{\omega(j)\left(1-k / e_{j}\right)} \prod_{p=0}^{k-1}\left(N-\log \varphi_{p}\left(H_{j}\right)\right)}\right]^{2 \beta_{k}} .
$$

Lemma 3.1. The function

$$
\frac{\left|\Lambda_{k}\right|}{\prod_{j=1}^{q}\left|\left(Z, A_{j}\right)\right|^{\omega(j)\left(1-k / e_{j}\right)}}
$$

is continuous on $\Delta_{R}$.

Proof. We shall prove that the function

$$
P=\left[\frac{\left|\Lambda_{k}\right|^{2}}{\prod_{j=1}^{q} \varphi_{0}\left(H_{j}\right)^{\omega(j)\left(1-k / e_{j}\right)}}\right]^{e}
$$

is continuous where $e=e_{1} \cdots e_{q}$. Lemma 3.1 follows from this. According to the expression of $P(z)$, we only need to consider the points at which $\left(Z, A_{j}\right)$ vanishes. For zero point $z_{0}$ of $\left(Z, A_{j}\right)$, since $f$ is ramified over $H_{j}$ with multiplicity at least $e_{j}$ for each $j$, we have

$$
\left(Z, A_{j}\right)=\left(z-z_{0}\right)^{\nu_{i}} Q_{j}(z)
$$

where $Q_{j}\left(z_{0}\right) \neq 0$, and $\nu_{j} \geq e_{j}$ or $\nu_{j}=0$. The $n$-subgeneral position implies that, at each point $z$, there are at most $n$ of hyperplanes $H_{j}$, such that $\left(Z(z), A_{j}\right)=0$. Thus there exists a constant $c_{0}$ (depending only on the given hyperplanes) such that

$$
\# B=\#\left\{j||\left(Z(z), A_{j}\right)|/| A_{j}|| Z(z) \mid \leq c_{0}\right\} \leq n .
$$

Let $E_{j}=1 / \varphi_{0}\left(H_{j}\right)^{\omega(j)\left(1-k / e_{j}\right)}$, then $E_{j} \leq 1$. If $j \notin B$, then $\varphi_{0}\left(H_{j}\right)>c_{0}$, so $E_{j} \leq c_{1}$ (depending only on the given hyperplanes).

Applying Lemma 2.5 with $E_{j}$ above, we obtain

$$
\begin{aligned}
\frac{\left|\Lambda_{k}\right|^{2}}{\prod_{j=1}^{q} \varphi_{0}\left(H_{j}\right)^{\omega(j)\left(1-k / e_{j}\right)}} & \leq c_{2} \frac{\left|\Lambda_{k}\right|^{2}}{\prod_{j \in B} \varphi_{0}\left(H_{j}\right)^{\omega(j)\left(1-k / e_{j}\right)}} \\
& \leq c_{2} \frac{\left|\Lambda_{k}\right|^{2}}{\prod_{j \in C} \varphi_{0}\left(H_{j}\right)^{\left(1-k / e_{j}\right)}} .
\end{aligned}
$$

We may assume the index set $C=\{1,2, \ldots, l\}$ and $l \leq k+1$, therefore

$$
\left[\prod_{j \in C}\left(Z(z), A_{j}\right)^{\left(1-k / e_{j}\right)}\right]^{e}=\left(z-z_{0}\right)^{b} R(z)
$$


where $b=\sum_{j=1}^{l} e \nu_{j}\left(1-k / e_{j}\right)$ and $R$ is a holomorphic function such that $R\left(z_{0}\right) \neq 0$. Since

$$
\begin{aligned}
\left|\Lambda_{k}\right| & =\operatorname{det}\left|\begin{array}{ccccc}
Z_{0} & Z_{1} & Z_{2} & \ldots & Z_{k} \\
Z_{0}^{\prime} & Z_{1}^{\prime} & Z_{2}^{\prime} & \cdots & Z_{k}^{\prime} \\
\vdots & \vdots & \vdots & & \vdots \\
Z_{0}^{(k)} & Z_{1}^{(k)} & Z_{2}^{(k)} & \ldots & Z_{k}^{(k)}
\end{array}\right| \\
& =\operatorname{det}\left|\begin{array}{cccc}
\left(Z, A_{1}\right) & \left(Z, A_{2}\right) & \left(Z, A_{3}\right) & \cdots \\
\left(Z, A_{1}\right)^{\prime} & \left(Z, A_{2}\right)^{\prime} & \left(Z, A_{3}\right)^{\prime} & \cdots \\
\vdots & \vdots & \vdots & \vdots \\
\left(Z, A_{1}\right)^{(k)} & \left(Z, A_{2}\right)^{(k)} & \left(Z, A_{3}\right)^{(k)} & \ldots
\end{array}\right|,
\end{aligned}
$$

we have $\Lambda_{k}=\left(z-z_{0}\right)^{\nu} S(z)$, where $\nu=\nu_{1}+\nu_{2}-1+\cdots+\nu_{l}-k$ and $S$ is a holomorphic function. Hence we obtain

$$
P(z) \leq\left|\left(z-z_{0}\right)^{2 p} T(z)\right|,
$$

where

$$
p=\frac{e k}{e_{1}}+\frac{e}{e_{2}}\left(k \nu_{2}-e_{2}\right)+\frac{e}{e_{3}}\left(k \nu_{3}-2 e_{3}\right)+\cdots+\frac{e}{e_{l}}\left(k \nu_{l}-(l-1) e_{l}\right) \geq 0,
$$

and $T(z)$ is continuous at $z_{0}$. Therefore $P(z)$ is bounded around $z_{0}$. Therefore $P(z)$ is continuous. Q.E.D.

Lemma 3.2. If $\sum_{j=1}^{q}\left(1-k / e_{j}\right) \geq 2 n-k+2$, and

$$
2 q / N<\left(\sum_{j=1}^{q} \omega(j)\left(1-k / e_{j}\right)-(k+1)\right) /\left(k^{2}+2 k\right),
$$

we have

(i) $\operatorname{Ric} \Gamma \geq \Gamma$ on $\Delta_{R}-\bigcup\left\{\varphi_{0}\left(H_{j}\right)=0\right\}$.

(ii) $\Gamma$ is a continuous pseudo-metric on $\Delta_{R}$.

Proof. From (3.3) and (3.4) it follows that

$$
\begin{aligned}
\operatorname{Ric} \Gamma= & -\beta_{k} \sum_{j=1}^{q} \omega(j)\left(1-\frac{k}{e_{j}}\right) d d^{c} \log \varphi_{0}\left(H_{j}\right) \\
& +\beta_{k} \sum_{j=1}^{q} \sum_{p=0}^{k-1} d d^{c} \log \left(1 /\left(N-\log \varphi_{p}\left(H_{j}\right)\right)\right)^{2} \\
& +\beta_{k} \sum_{p=0}^{k-1}\left(1 / \lambda_{p}\right) \operatorname{Ric} \Omega_{p} .
\end{aligned}
$$

By Lemma 2.1, (2.2), and that $d d^{c} \log \varphi_{0}\left(H_{j}\right)=-\Omega_{0}$, we have

$$
\begin{aligned}
\operatorname{Ric} \Gamma \geq \beta_{k}( & \sum_{j=1}^{q} \omega(j)\left(1-\frac{k}{e_{j}}\right) \Omega_{0}+2 \sum_{j=1}^{q} \sum_{p=0}^{k-1} \frac{\varphi_{p+1}\left(H_{j}\right)}{\varphi_{p}\left(H_{j}\right)\left(N-\log \varphi_{p}\left(H_{j}\right)\right)^{2}} \Omega_{p} \\
& \left.-\frac{2 q}{N} \sum_{p=0}^{k-1} \Omega_{p}+\sum_{p=0}^{k-1}\left[(k-p)+(k-p)^{2} \frac{2 q}{N}\right]\left\{\Omega_{p+1}-2 \Omega_{p}+\Omega_{p-1}\right\}\right) .
\end{aligned}
$$


Using Lemma 2.6, we obtain

$$
\begin{aligned}
& \sum_{j=1}^{q} \frac{\varphi_{p+1}\left(H_{j}\right)}{\varphi_{p}\left(H_{j}\right)\left(N-\log \varphi_{p}\left(H_{j}\right)\right)^{2}} \Omega_{p} \\
& \quad \leq c_{p}\left[\prod_{j=1}^{q}\left(\frac{\varphi_{p+1}\left(H_{j}\right)}{\varphi_{p}\left(H_{j}\right)}\right)^{\omega(j)} \frac{1}{\left(N-\log \varphi_{p}\left(H_{j}\right)\right)^{2}}\right]^{\lambda_{p}} \Omega_{p} \\
& \quad \geq \frac{i}{2 \pi} \sigma_{p} d z \wedge d \bar{z} .
\end{aligned}
$$

We also notice that $\Omega_{k}=0$ so that

$$
\sum_{p=0}^{k-1}(k-p)\left(\Omega_{p+1}-2 \Omega_{p}+\Omega_{p-1}\right)=-(k+1) \Omega_{0}
$$

and therefore

$$
\begin{aligned}
\operatorname{Ric} \Gamma \geq \beta_{k}\left(\sum_{j=1}^{q} \omega(j)\right. & \left(1-\frac{k}{e_{j}}\right) \Omega_{0}+2 \frac{i}{2 \pi} \sum_{p=0}^{k-1} \sigma_{p} d z \wedge d \bar{z}-(k+1) \Omega_{0}-\left(k^{2}+2 k\right) \frac{2 q}{N} \Omega_{0} \\
+ & \left.\sum_{p=1}^{k-2}\left[(k-p+1)^{2}-2(k-p)^{2}+(k-p-1)^{2}-1\right] \frac{2 q}{N} \Omega_{p}+\frac{2 q}{N} \Omega_{k-1}\right) .
\end{aligned}
$$

The following is an elementary inequality:

For all the positive numbers $x_{1}, \ldots, x_{n}$ and $a_{1}, \ldots, a_{n}$,

$$
a_{1} x_{1}+\cdots+a_{n} x_{n} \geq\left(a_{1}+\cdots+a_{n}\right)\left(x_{1}^{a_{1}} \cdots x_{n}^{a_{n}}\right)^{1 /\left(a_{1}+\cdots+a_{n}\right)} .
$$

Letting $a_{p}=\lambda_{p}^{-1}$ in (3.6), we have

$$
\sum_{p=0}^{k-1} \sigma_{p} \geq \frac{c}{2 \beta_{k}} \sum_{p=0}^{k-1} \sigma_{p}^{\beta_{k} / \lambda_{p}}
$$

and therefore

$$
\begin{aligned}
\operatorname{Ric} \Gamma \geq \beta_{k}\left(\left(\sum_{j=1}^{q} \omega(j)\left(1-\frac{k}{e_{j}}\right)-(k+1)\right.\right. & \left.-\left(k^{2}+2 k\right) \frac{2 q}{N}\right) \Omega_{0} \\
& \left.+\sum_{p=0}^{k-2} \frac{2 q}{N} \Omega_{p}+\frac{2 q}{N} \Omega_{k-1}\right)+\Gamma .
\end{aligned}
$$

By Lemma 2.2, we find

$$
\begin{aligned}
& \theta\left(\sum_{j=1}^{q} \omega(j)\left(1-\frac{k}{e_{j}}\right)-k-1\right)=\theta\left(\sum_{j=1}^{q} \omega(j)-k-1\right)-\frac{\sum_{j=1}^{q} \omega(j) \theta k}{e_{j}} \\
& \quad=q-2 n+k-1-\frac{\sum_{j=1}^{q} \omega(j) \theta k}{e_{j}} \geq q-2 n+k-1-\frac{k}{e_{j}} \\
& \quad=\sum_{j=1}^{q}\left(1-\frac{k}{e_{j}}\right)-2 n+k-1>0
\end{aligned}
$$


and $\theta>0$, so

$$
\sum_{j=1}^{q} \omega(j)\left(1-\frac{k}{e_{j}}\right)-(k+1)>0 .
$$

This implies $\operatorname{Ric} \Gamma \geq \Gamma$. Thus (i) is satisfied.

(ii) follows from Lemma 3.1, (3.3) and (3.5). Q.E.D.

We recall the following generalization of the Schwarz lemma.

Lemma 3.3. Let $\Gamma=\frac{i}{2 \pi} h(z) d z \wedge d \bar{z}$ be a continuous pseudo-metric on $\Delta_{R}$ whose curvature is bounded above by a negative constant. Then, for some positive $c_{0}, h(z) \leq c_{0}\left(2 R /\left(R^{2}-|z|^{2}\right)\right)^{2}$. For the proof, see [1, pp. 12-14].

The purpose of this section is to obtain the following lemma.

Main Lemma. Let $f=\left[Z_{0}: \cdots: Z_{k}\right]: \Delta_{R} \rightarrow C P^{k}$ be a nondegenerate holomorphic map, $H_{1}, \ldots, H_{q}$ be hyperplanes in $C P^{k}$ in $n$-subgeneral position, $\omega(j)$ be their Nochka weights. Let $H_{j}:\left(W, A_{j}\right)=0$ and $Z=\left(Z_{0}, \ldots, Z_{k}\right)$. If $f$ is ramified over $H_{j}$ with multiplicity at least $e_{j}$ for each $j, \sum_{j=1}^{q}\left(1-k / e_{j}\right)>$ $2 n-k+1$ and $N>2 q\left(k^{2}+2 k\right) /\left(\sum_{j=1}^{q} \omega(j)\left(1-k / e_{j}\right)-(k+1)\right)$, then there exists a positive constant $c$ such that

$$
\begin{aligned}
& |Z|^{\sum_{j=1}^{q} \omega(j)\left(1-k / e_{j}\right)-(k+1)-\left(k^{2}+2 k-1\right) 2 q / N} \frac{\prod_{p=0}^{k-1} \prod_{j=1}^{q}\left|\Lambda_{p} \vee A_{j}\right|^{4 / N}\left|\Lambda_{k}\right|^{1+2 q+N}}{\prod_{j=1}^{q}\left|\left(Z, A_{j}\right)\right|^{\omega(j)\left(1-k / e_{j}\right)}} \\
& \quad \leq c\left(\frac{2 R}{\left(R^{2}-|z|^{2}\right)}\right)^{k(k+1) 2+\sum_{p=0}^{k-1}(k-p)^{2} 2 q / N}
\end{aligned}
$$

Proof. Using the above Schwarz lemma for $\Gamma$, we obtain

$$
h(z) \leq c_{0}\left(2 R /\left(R^{2}-|z|^{2}\right)\right)^{2} .
$$

So by (3.5) we have

$$
\begin{aligned}
& |Z|^{\sum_{j=1}^{q} \omega(j)\left(1-k / e_{j}\right)-(k+1)-\left(k^{2}+2 k-1\right) 2 q / N} \frac{\left(\left|\Lambda_{1}\right| \cdots \mid \Lambda_{k-1}\right)^{4 q / N}\left|\Lambda_{k}\right|^{1+2 q / N}}{\prod_{j=1}^{q}\left|\left(Z, A_{j}\right)\right|^{\omega(j)\left(1-k / e_{j}\right)} \prod_{p=0}^{k-1}\left(N-\log \varphi_{p}\left(H_{j}\right)\right)} \\
& \quad \leq c_{0}\left(\frac{2 R}{R^{2}-|z|^{2}}\right)^{1 / \beta_{k}} .
\end{aligned}
$$
have

Set $K:=\sup _{0<x \leq 1} x^{2 / N}(N-\log x)$. Since $\varphi_{p}\left(H_{j}\right)<1$ for all $p$ and $j$ we

$$
\frac{1}{\left(N-\log \varphi_{p}\left(H_{j}\right)\right)} \geq \frac{1}{K} \varphi_{p}\left(H_{j}\right)^{2 / N}=\frac{1}{K} \frac{\left|\Lambda_{p} \vee A_{j}\right|^{4 / N}}{\left|\Lambda_{p}\right|^{4 / N}} .
$$

Substituting these into (3.7), we obtain the desired conclusion.

\section{Proof OF THEOREM 1}

The proof of Theorem 1 basically follows the argument in [13] using the main lemma (see also the arguments in [6,7 and 8]). We include our proof here for the convenience of the reader.

We may assume $M$ is simply connected, otherwise we consider its universal covering. By Koebe's uniformization theorem, $M$ is bioholomorphic to $C$ or to the unit disc. For the case $M=C$, Nochka (see [10], also see [16]) proved 
that if a $k$-nondegenerate holomorphic map from $C$ to $C P^{n-1}$ is ramified over hyperplanes $H_{j} \quad(1 \leq j \leq q)$ with multiplicity at least $e_{j}$, where $H_{j}$ are in general position, then

$$
\sum_{j=1}^{q}\left(1-\frac{k}{e_{j}}\right) \leq 2(n-1)-k+1
$$

in this case our Theorem 1 is true. For our purpose it suffices to consider the case $M=\Delta$.

We first prove the first part of Theorem 1.

Assume the first part of Theorem 1 is not true, namely $G$ is ramified over hyperplanes $H_{1}, \ldots, H_{q}$ in $C P^{n-1}$ in general position with multiplicity $e_{j}$ and

$$
\sum_{j=1}^{q}\left(1-k / e_{j}\right)>(k+1)(n-k / 2-1)+n .
$$

Let $\omega(j)$ be Nochka weights of $\left\{H_{j}\right\}$. Because $G$ is $k$-nondegenerate, we may assume $G(\Delta) \subset C P^{k}$, so that $G=\left[g_{0}: \cdots: g_{k}\right]: \Delta \rightarrow C P^{k}$ is nondegenerate. We consider hyperplanes $H_{j} \cap C P^{k}$, obviously these hyperplanes are in $(n-1)$-subgeneral position in $C P^{k}$. For the convenience, we still denote these hyperplanes by $\left\{H_{j}\right\}$.

Let $\widetilde{G}=\left(g_{0}, \ldots, g_{k}\right): \Delta \rightarrow C P^{k+1}-\{0\}$; then the metric $d s^{2}$ on $M$ induced from the standard metric on $R^{n}$ is given by

$$
d s^{2}=2|\widetilde{G}|^{2}|d z|^{2} .
$$

By Lemma 2.2,

$$
q-2(n-1)+k-1=\theta\left(\sum_{j=1}^{q} \omega(j)-k-1\right), \quad 0<\omega(j) \theta \leq 1,
$$

and

so

$$
\theta \leq \frac{2(n-1)-k+1}{k+1}=\frac{2 n-k-1}{k+1}
$$

$$
\begin{aligned}
2\left(\sum_{j=1}^{q} \omega(j)\left(1-\frac{k}{e_{j}}\right)-k-1\right) & =\frac{2 \theta\left(\sum_{j=1}^{q} \omega(j)-k-1\right)}{\theta}-2 \sum_{j=1}^{q} \frac{k \omega(j) \theta}{\theta e_{j}} \\
& =\frac{2(q-2 n+k+1)}{\theta}-2 \sum_{j=1}^{q} \frac{k \omega(j) \theta}{\theta e_{j}} \\
& \geq \frac{2(q-2 n+k+1)}{\theta}-2 \sum_{j=1}^{q} \frac{k}{\theta e_{j}} \\
& =\frac{2\left(\sum_{j=1}^{q}\left(1-k / e_{j}\right)-2 n+k+1\right)}{\theta} \\
& \geq \frac{2\left(\sum_{j=1}^{q}\left(1-k / e_{j}\right)-2 n+k+1\right)(k+1)}{(2 n-k-1)} \\
& >k(k+1) \quad(\text { by }(4.1)) .
\end{aligned}
$$


Consider numbers

$$
\begin{gathered}
\rho=\frac{k(k+1) / 2+\sum_{p=0}^{k-1}(k-p)^{2} 2 q / N}{\sum_{j=1}^{q} \omega(j)\left(1-k / e_{j}\right)-(k+1)-\left(k^{2}+2 k-1\right) 2 q / N} \\
\gamma=\frac{k(k+1) / 2+q k(k+1) / N+2 q / N \sum_{p=0}^{k-1} p(p+1)}{\sum_{j=1}^{q} \omega(j)\left(1-k / e_{j}\right)-(k+1)-\left(k^{2}+2 k-1\right) 2 q / N} \\
\delta=\frac{1}{(1-\gamma)\left(\sum_{j=1}^{q} \omega(j)\left(1-k / e_{j}\right)-(k+1)-\left(k^{2}+2 k-1\right) 2 q / N\right)} .
\end{gathered}
$$

Choose some $N$ with

$$
\begin{aligned}
& \frac{\sum_{j=1}^{q} \omega(j)\left(1-k / e_{j}\right)-(k+1)-k(k+1) / 2}{k^{2}+2 k-1+\sum_{p=0}^{k}(k-p)^{2}} \\
&>2 q / N>\frac{\sum_{j=1}^{q} \omega(j)\left(1-k / e_{j}\right)-(k+1)-k(k+1) / 2}{1 / q+\left(k^{2}+2 k-1\right)+k(k+1) / 2+\sum_{p=0}^{k-1} p(p+1)}
\end{aligned}
$$

so that

$$
0<\rho<1,2 \delta / N>1 .
$$

Consider the open subset

$$
M^{\prime}=M-\left(\left\{\widetilde{G}_{k}=0\right\} \bigcup_{1 \leq j \leq q, 0 \leq p \leq k-1}\left\{\widetilde{G}_{p} \vee A_{j}=0\right\}\right)
$$

of $M$ and define the function

$$
v=\left(\frac{\prod_{j=1}^{q}\left|\left(G, A_{j}\right)\right|^{\omega(j)\left(1-k / e_{j}\right)}}{\prod_{p=0}^{k-1} \prod_{j=1}^{q}\left|\widetilde{G}_{p} \vee A_{j}\right|^{4 / N}\left|\widetilde{G}_{k}\right|^{1+2 q / N}}\right)^{\delta}
$$

on $M^{\prime}$, where $\widetilde{G}_{p}=\widetilde{G}^{(0)} \wedge \cdots \wedge \widetilde{G}^{(p)}$. By Lemma $3.1, \nu(z)$ is strictly positive and continuous on $M^{\prime}$.

Let $\pi: \widetilde{M}^{\prime} \rightarrow M^{\prime}$ be the universal covering of $M^{\prime}$. Since $\log v \circ \pi$ is harmonic on $\widetilde{M}^{\prime}$ by the assumption, we can take a holomorphic function $\beta$ on $\widetilde{M}^{\prime}$ such that $|\beta|=v \circ \pi$. Without loss of generality, we may assume that $M^{\prime}$ contains the origin 0 of $C$. As in Fujimoto's paper [6, 7, 8], for each point $\widetilde{p}$ of $\widetilde{M}^{\prime}$ we take a continuous curve $\gamma_{\tilde{p}}:[0,1] \rightarrow M^{\prime}$ with $\gamma_{\tilde{p}}(0)=0$ and $\gamma_{\tilde{p}}(1)=\pi(\tilde{p})$, which corresponds to the homotopy class of $\tilde{p}$. Let $\tilde{0}$ denote the point corresponding to the constant curve 0 . Set

$$
w=F(\tilde{p})=\int_{\gamma_{\tilde{p}}} \beta(z) d z .
$$

Then $F$ is a single-valued holomorphic function on $M^{\prime}$ satisfying the condition $F(\tilde{0})=0$ and $d F(\tilde{p}) \neq 0$ for every $\tilde{p} \in \widetilde{M}^{\prime}$. Choose the largest $R(\leq \infty)$ such that $F$ maps an open neighborhood $U$ of 0 biholomorphically onto an open disc $\Delta_{R}$ in $C$, and consider the map $B=\pi \circ(F \mid U)^{-1}: \Delta_{R} \rightarrow M^{\prime}$. By the Liouville theorem, $R=\infty$ is impossible. 
By the definition of $w=F(z)$ we have

$$
|d w / d z|=v(z) \text {. }
$$

For each point $a \in \partial \Delta$ consider the curve

$$
L_{a}: w=t a, \quad 0 \leq t<1,
$$

and the image $\Gamma_{a}$ of $L_{a}$ by $B$. We shall show that there exists a point $a_{0}$ in $\partial \Delta_{R}$ such that $\Gamma_{a_{0}}$ tends to the boundary of $M$. To this end, we assume the contrary. Then, for each $a \in \partial \Delta_{R}$, there is a sequence $\left\{t_{\nu}: \nu=1,2, \ldots\right\}$ such that $\lim _{\nu \rightarrow \infty} t_{\nu}=1$ and $z_{0}=\lim _{\nu \rightarrow \infty} B\left(t_{\nu} a\right)$ exist in $M$. Suppose that $z_{0} \notin M^{\prime}$. Let $\delta_{0}=4 \delta / N>1$. Then by Lemma 3.1, we have

$$
\liminf _{z \rightarrow z_{0}}\left|\widetilde{G}_{k}\right|^{\delta_{0}} \prod_{1 \leq j \leq q,}\left|\widetilde{G}_{p} \vee A_{j}\right|^{2 \delta_{0}} \cdot v>0 .
$$

If $\widetilde{G}_{k}\left(z_{0}\right)=0$ or $\left|\widetilde{G}_{p} \vee A_{j}\right|\left(z_{0}\right)=0$ for some $p$ and $j$, we can find a positive constant $c$ such that $v \geq c /\left|z-z_{0}\right|^{\delta_{0}}$ in a neighborhood of $z_{0}$, so that we obtain

$$
\begin{aligned}
R & =\int_{L_{a}}|d w|=\int_{L_{a}}\left|\frac{d w}{d z}\right||d z|=\int v(z)|d z| \\
& \geq c \int_{\Gamma} \frac{1}{\left|z-z_{0}\right|^{\delta_{0}}}|d z|=\infty .
\end{aligned}
$$

This is a contradiction. Therefore, we have $z_{0} \in M^{\prime}$.

Take a simply connected neighborhood $V$ of $z_{0}$ which is relatively compact in $M^{\prime}$. Set $C^{\prime}=\min _{z \in V} v(z)>0$. Then $B(t a) \in V \quad\left(t_{0}<t<1\right)$ for some $t_{0}$. In fact, if not, $\Gamma_{a}$ goes and returns infinitely often from $\partial V$ to a sufficiently small neighborhood of $z_{0}$ and so we get the absurd conclusion

$$
R=\int_{L_{a}}|d w| \geq c^{\prime} \int_{\Gamma_{a}}|d z|=\infty .
$$

By the same argument, we can easily see that $\lim _{t \rightarrow 1} B(t a)=z_{0}$. Since $\pi$ maps each connected component of $\pi^{-1}(V)$ bioholomorphically onto $V$, there exists the limit

$$
\tilde{p}_{0}=\lim _{t \rightarrow 1}(F \mid U)^{-1}(t a) \in \widetilde{M}^{\prime} .
$$

Thus $(F \mid U)^{-1}$ has a biholomorphic extension to a neighborhood of $a$. Since $a$ is arbitrarily chosen, $F$ maps an open neighborhood of $\bar{U}$ biholomorphically onto an open neighborhood of $\bar{\Delta}_{R}$. This contradicts the property of $R$. In conclusion, there exists a point $a_{0} \in \partial \Delta_{R}$ such that $\Gamma_{a_{0}}$ tends to the boundary of $M$.

Our goal is to show that $\Gamma_{a_{0}}$ has finite length, contradicting the completeness of the given minimal surface $M$.

By (4.7) we obtain $|d w / d z|=v(z)$. So

$$
\begin{aligned}
\left|\frac{d w}{d z}\right| & =|v(z)|^{1-\gamma}\left|\frac{d w}{d z}\right|^{\gamma} \\
& =\left(\frac{\prod_{j=1}^{q}\left|\left(\widetilde{G}, A_{j}\right)\right|^{\omega(j)\left(1-k / e_{j}\right)}}{\prod_{p=0}^{k-1} \prod_{j=1}^{q}\left|\widetilde{G}_{p} \vee A_{j}\right|^{4 / N}\left|\widetilde{G}_{k}\right|^{1+2 q / N}}\right)^{1 /\left(\sum \omega(j)\left(1-k / e_{j}\right)-(k+1)-\left(k^{2}+2 k-1\right) 2 q / N\right)}\left|\frac{d w}{d z}\right|^{\gamma} .
\end{aligned}
$$


Let $Z(w)=\widetilde{G} \circ B(w), Z_{0}(w)=g_{0} \circ B(w), \ldots, Z_{k}(w)=g_{k} \circ B(w)$. Then because

$$
Z \wedge Z^{\prime} \wedge \cdots \wedge Z^{(p)}=\left(\widetilde{G} \wedge \cdots \wedge \widetilde{G}^{(p)}\right)\left(\frac{d z}{d w}\right)^{p(p+1) / 2},
$$

it is easy to see that

$$
\left|\frac{d w}{d z}\right|=\left(\frac{\prod_{j=1}^{p}\left|\left(Z, A_{j}\right)\right|^{\omega(j)\left(1-k / e_{j}\right)}}{\prod_{p=0}^{k-1} \prod_{j=1}^{q}\left|\Lambda_{p} \vee A_{j}\right|^{4 / N}\left|\Lambda_{k}\right|^{1+2 q / N}}\right)^{1 /\left(\sum \omega(j)\left(1-k / e_{j}\right)-(k+1)-\left(k^{2}+2 k-1\right) 2 q / N\right)},
$$

where $\Lambda_{p}=Z^{(0)} \wedge \cdots \wedge Z^{(p)}$.

On the other hand, the metric on $\Delta_{R}$ induced from $d s^{2}=2|\widetilde{G}|^{2}|d z|^{2}$ through $B$ is given by

$$
B^{*} d s^{2}=2|\widetilde{G}(B(w))|^{2}\left|\frac{d z}{d w}\right|^{2}|d w|^{2} .
$$

Combining (4.7) and (4.8) gives

$$
B^{*} d s=2|Z|\left(\frac{\prod_{p=0}^{k-1} \prod_{j=1}^{q}\left|\Lambda_{p} \vee A_{j}\right|^{4 / N}\left|\Lambda_{k}\right|^{1+2 q / N}}{\prod_{j=1}^{q}\left|\left(Z, A_{j}\right)\right|^{\omega(j)\left(1-k / e_{j}\right)}}\right)^{1 /\left(\sum \omega(j)\left(1-k / e_{j}\right)-(k+1)-\left(k^{2}+2 k-1\right) 2 q / N\right)}|d w| .
$$

Using the main lemma, we have

$$
B^{*} d s \leq c\left(\frac{2 R}{R^{2}-|w|^{2}}\right)^{\rho}|d w|,
$$

where $c$ is a positive constant. Since $\rho<1$, it then follows that

$$
d(0) \leq \int_{\Gamma_{a_{0}}} d s=\int_{L_{a_{0}}} B^{*} d s \leq c \int_{0}^{R}\left(\frac{2 R}{R^{2}-|w|^{2}}\right)^{\rho}|d w|<\infty,
$$

where $d(0)$ denotes the distance from the origin 0 to the boundary of $M$. This contradicts the assumption of completeness of $M$. Hence the proof of the first part of Theorem 1 is complete.

We now prove the second part.

For any complete minimal surface $M$ immersed in $R^{n}$, if there are $q>$ $n(n+1) / 2$ hyperplanes in general position in $C P^{n-1}$ such that its Gauss map $G$ is ramified over $H_{j}$ with multiplicity at least $e_{j}$ for each $j$ and

$$
\sum_{j=1}^{q}\left(1-n / e_{j}\right)>n(n+1) / 2,
$$

we are going to prove that $M$ is flat. Since $M$ is flat if and only if its Gauss map is a constant map (see [12]), we only need to prove that $G$ is a constant map.

If $G$ is not a constant map, then we may assume that $G$ is $k$-nondegenerate and $1 \leq k \leq n-1$. By the first part of the theorem, we have

$$
\sum_{j=1}^{q}\left(1-k / e_{j}\right) \leq(k+1)(n-k / 2-1)+n \text {. }
$$

Since

$$
(k+1)(n-k / 2-1)+n \leq n(n+1) / 2,
$$


and

$$
\sum_{j=1}^{q}\left(1-(n-1) / e_{j}\right) \leq \sum_{j=1}^{q}\left(1-k / e_{j}\right)
$$

we obtain

$$
\sum_{j=1}^{q}\left(1-(n-1) / e_{j}\right) \leq n(n+1) / 2 .
$$

This contradicts the assumption. Therefore $M$ is flat. Q.E.D.

\section{Proof OF THEOREM 2}

Let $x=\left(x_{1}, x_{2}, x_{3}\right): M \rightarrow R^{3}$ be a nonflat minimal surface and $g: M \rightarrow$ $C P^{1}$ the Gauss map. Assume $M=\Delta$ (as the argument above). Set $\varphi_{i}=$ $\partial x_{i} / \partial z(i=1,2,3)$ and $f=\varphi_{1}-\sqrt{-1} \varphi_{2}$. Then according to [12] or [7], the metric on $M$ induced from $R^{3}$ is given by

$$
d s^{2}=|f|^{2}\left(1+|g|^{2}\right)^{2}|d z|^{2} .
$$

Take a reduced representation $\tilde{g}=\left(g_{0}, g_{1}\right)$ of $g$ on $M$. Then we can rewrite

$$
d s^{2}=|h|^{2}|\tilde{g}|^{4}|d z|^{2},
$$

where $h=f / g_{0}^{2}$, and moreover $h \neq 0$. The rest of the steps are the same as the proof of Theorem 1. If $M$ is not flat, then $g$ is not a constant map. Assume that $g$ is ramified over $a_{j}$ with multiplicity of $e_{j}$ and $\sum_{j=1}^{q}\left(1-1 / e_{j}\right)>4$, we shall derive a contradiction. Let $P\left(\alpha_{j}\right)=a_{j}, \alpha_{j} \in C^{2}$. Consider numbers

$$
\begin{aligned}
& \rho=\gamma=\frac{1+2 q / N}{\sum_{j=1}^{q}\left(1-1 / e_{j}\right)-2-2 q / N}, \\
& \delta=\frac{1}{(1-\rho)\left(\sum_{j=1}^{q}\left(1-1 / e_{j}\right)-2-2 q / N\right)} .
\end{aligned}
$$

Choose some $N$ with

$$
\frac{\sum_{j=1}^{q}\left(1-1 / e_{j}\right)-3}{3}>2 q / N>\frac{\sum_{j=1}^{q}\left(1-1 / e_{j}\right)-3}{3+1 / q}
$$

so that $0<2 \rho<1, \frac{2 \delta}{N}>1$. Consider the open subset $M^{\prime}=M-\left(\left\{\tilde{g}_{1}=0\right\}\right)$ of $M$ and define the function

$$
v=h^{1 /(1-\gamma)}\left(\frac{\prod_{j=1}^{q}\left|\left(\tilde{g}, \alpha_{j}\right)\right|^{\left(1-1 / e_{j}-4 / N\right)}}{\left|\tilde{g}_{1}\right|^{1+2 q / N}}\right)^{\delta}
$$

on $M^{\prime}$ where $\tilde{g}_{1}=\tilde{g} \wedge \tilde{g}^{\prime}$.

By exactly the same argument as in the proof of Theorem 1, we can find a curve $\Gamma_{a_{0}}$ tends to the boundary of $M$, and we can estimate the pull-back metric, eventually we obtain that $\Gamma_{a_{0}}$ has finite length, contradicting the completeness of the given minimal surface $M$. Q.E.D. 


\section{REFERENCES}

1. L. A. Ahlfors, Conformal invariants, Topics in Geometric Function Theory, McGraw-Hill, New York, 1973.

2. W. Chen, Defect relations for degenerate meromorphic maps, Trans. Amer. Math. Soc. 319 (1990), 499-515.

3. S. S. Chern and R. Osserman, Complete minimal surfaces in euclidean $n$-space, J. Analyse Math. 19 (1967), 15-34.

4. M. J. Cowen, The Kobayashi metric on $P^{n}-\left(2^{n}+1\right)$ hyperplanes, Value Distribution Theory, Marcel Dekker, New York, 1974, pp. 205-223.

5. M. J. Cowen and P. A. Griffiths, Holomorphic curves and metrics of negative curvature, J. Analyse Math. 29 (1976), 93-153.

6. H. Fujimoto, On the Gauss map of a complete minimal surface in $R^{m}$, J. Math. Soc. Japan 35 (1983), 279-288.

7. _ On the number of exceptional values of the Gauss map of minimal surfaces, J. Math. Soc. Japan 49 (1988), 235-247.

8. _ _ Modified defect relations for the Gauss map of minimal surfaces. II, J. Differential Geom. 31 (1990), 365-385.

9. X. Mo and R. Osserman, On the Gauss map and total curvature of complete minimal surfaces and an extension of Fujimoto's theorem, J. Differential Geom. 31 (1990), 343-355.

10. E. I. Nochka, Uniqueness theorems for rational functions on algebraic varieties, Bul. Akad. Shtiintsa RSS Moldoven 3 (1979), 27-31. (Russian)

11. __ On the theory of meromorphic functions, Soviet Math. Dokl. 27 (1983), no. 2.

12. R. Osserman, A survey of minimal surfaces, 2nd ed., Dover, New York, 1986.

13. $\mathrm{M}$. Ru, On the Gauss map of minimal surfaces immersed in $R^{n}$, J. Differential Geom. 34 (1991), 411-423.

14. __, On the Gauss map of minimal surfaces with finite total curvature, Bull. Austral. Math. Soc. 44 (1991), 225-232.

15. M. Ru and P. M. Wong, Integral points of $P^{n}-\{2 n+1$ hyperplanes in general position $\}$, Invent. Math. 106 (1991), 195-216.

16. F. Sakai, Degeneracy of holomorphic maps with ramification, Invent. Math. 26 (1974), 213229.

17. B. V. Shabat, Distribution of values of holomorphic mappings, Transl. Math. Monographs, vol. 61, Amer. Math. Soc., Providence, R.I., 1985.

18. P. M. Wong, Defect relations for maps on parabolic spaces and Kobayashi metric on projective spaces omitting hyperplanes, Thesis, Univ. of Notre Dame, 1976.

19. F. Xavier, The Gauss map of a complete non-flat minimal surfaces cannot omit 7 points of the sphere, Ann. of Math. (2) 113 (1981), 211-214; Erratum, Ann. of Math. (2) 115 (1985), 667.

Department of Mathematics, National University of Singapore, Singapore 0511, REPUBLIC OF SINGAPORE 02138

Current address: Department of Mathematics, Harvard University, Cambridge, Massachusetts

E-mail address: minru@math.harvard.edu 